\title{
Article
}

Subscriber access provided by UNIV OF TECH SYDNEY

\section{Application of Targeted Molecular and Material Property Optimization to Bacterial Attachment-Resistant (Meth)acrylate Polymers}

Kevin Adlington, Nam Trong Nguyen, Elizabeth Eaves, jing yang, CHIEN YI CHANG, Jianing Li, Alexandra L. Gower, Amy Stimpson, Daniel G. Anderson, Robert Langer, Martyn C. Davies, Andrew L. Hook, Paul Williams, Morgan Russell Alexander, and Derek J Irvine

Biomacromolecules, Just Accepted Manuscript • DOI: 10.1021/acs.biomac.6b00615 • Publication Date (Web): 26 Jul 2016

Downloaded from http://pubs.acs.org on July 27, 2016

\section{Just Accepted}

"Just Accepted" manuscripts have been peer-reviewed and accepted for publication. They are posted online prior to technical editing, formatting for publication and author proofing. The American Chemical Society provides "Just Accepted" as a free service to the research community to expedite the dissemination of scientific material as soon as possible after acceptance. "Just Accepted" manuscripts appear in full in PDF format accompanied by an HTML abstract. "Just Accepted" manuscripts have been fully peer reviewed, but should not be considered the official version of record. They are accessible to all readers and citable by the Digital Object Identifier (DOI®). "Just Accepted" is an optional service offered to authors. Therefore, the "Just Accepted" Web site may not include all articles that will be published in the journal. After a manuscript is technically edited and formatted, it will be removed from the "Just Accepted" Web site and published as an ASAP article. Note that technical editing may introduce minor changes to the manuscript text and/or graphics which could affect content, and all legal disclaimers and ethical guidelines that apply to the journal pertain. ACS cannot be held responsible for errors or consequences arising from the use of information contained in these "Just Accepted" manuscripts. 


\title{
Application of Targeted Molecular and Material Property Optimization to Bacterial Attachment-Resistant (Meth)acrylate Polymers
}

\author{
Kevin Adlington, ${ }^{a}$ Nam T. Nguyen, ${ }^{a, b}$ Elizabeth Eaves, ${ }^{\text {a,b }}$ Jing Yang, ${ }^{c}$ Chien-Yi Chang,,${ }^{c, d}$ \\ Jianing Li, ${ }^{\mathrm{a}, \mathrm{b}}$ Alexandra L. Gower, ${ }^{\mathrm{a}}$ Amy Stimpson, ${ }^{\mathrm{a}}$ Daniel G. Anderson, ${ }^{\mathrm{e}}$ Robert Langer, ${ }^{\mathrm{e}}$ \\ Martyn C. Davies, ${ }^{\mathrm{f}}$ Andrew L. Hook, ${ }^{\mathrm{f}}$ Paul Williams, ${ }^{\mathrm{c}}$ Morgan R. Alexander, ${ }^{* *}$ and Derek J. \\ Irvine $^{\mathrm{a}^{*}}$ \\ ${ }^{a}$ Department of Chemical and Environmental Engineering, Faculty of Engineering, \\ University of Nottingham, Nottingham, NG7 2RD, UK. \\ ${ }^{\mathrm{b}}$ School of Chemistry, University of Nottingham, Nottingham, NG7 2RD, UK. \\ ${ }^{\mathrm{c}}$ Centre for Biomolecular Sciences and School of Life Sciences, School of Chemistry, \\ University of Nottingham, Nottingham, NG7 2RD, UK. \\ ${ }^{\mathrm{d}}$ Current address - School of Life Sciences, Heriot-Watt University, Edinburgh, EH14 4AS, \\ UK. \\ ${ }^{\mathrm{e}}$ Department of Chemical Engineering, Institute for Medical Engineering and Science, \\ Harvard-MIT Division of Health Sciences and Technology, David H. Koch Institute for \\ Integrative Cancer Research, Massachusetts Institute of Technology, 500 Main Street, \\ Cambridge, MA, 02139, USA. \\ ${ }^{\mathrm{f}}$ Laboratory of Biophysics and Surface Analysis, School of Pharmacy, University of \\ Nottingham, Nottingham, NG7 2RD, UK.
}

\begin{abstract}
Developing medical devices that resist bacterial attachment and subsequent biofilm formation is highly desirable. In this paper, we report the optimization of the molecular structure and thus material properties of a range of (meth)acrylate copolymers which contain monomers reported to deliver bacterial resistance to surfaces. This optimization allows such monomers to be employed within novel coatings to reduce bacterial attachment to silicone urinary catheters. We show that the flexibility of copolymers can be tuned to match that of the silicone catheter substrate, by copolymerizing these polymers with a lower $\mathrm{T}_{\mathrm{g}}$ monomer such that it passes the flexing fatigue tests as coatings upon catheters, that the homopolymers failed. Furthermore, the $T_{g}$ values of the copolymers are shown to be readily estimated by the Fox equation. The bacterial resistance performance of these copolymers were typically found to be better than the neat silicone or a commercial silver containing hydrogel surface, when the monomer feed contained only $25 \mathrm{v} \%$ of the "hit" monomer. The method of initiation (either photo or thermal) was shown not to affect the bacterial resistance of the copolymers.
\end{abstract}


Optimized synthesis conditions to ensure that the correct copolymer composition and prevent the onset of gelation are detailed.

\section{Introduction}

Biofouling, or the formation of biofilms on the surfaces of devices/implants/manufactured structures, represents a major problem for the application of such constructs in industrial/market sectors such as medical implantation, healthcare devices, aerospace, marine structures, oil recovery and in manufacturing industry. ${ }^{1}$ The formation of biofilms is hugely undesirable as can lead to many negative effects during device use/application, from the development of infection or irritation in medical or healthcare applications through to increased fuel demand in transportation uses due to increased drag and blockage/erosion of pipes in manufacturing plants. Consequently, the development of novel biofouling-resistant materials or coatings which outperform existing commercial benchmark materials will deliver enormous economic and societal benefits.

In the particular area of medical devices, the presence of bacterial biofilms (i.e. surface attached communities of bacterial cells embedded in a self-generated exopolymer matrix) leads to greater persistence, since bacteria within biofilms exhibit up to 1000 times greater resistance to the action of both antimicrobial additives/agents and the host's natural immune defenses than their free-living (planktonic) counterparts. ${ }^{1 b, 2}$ The current methods for reducing biofilm associated infections typically focus on the modification of the materials used to manufacture in-dwelling medical devices via the incorporation of antibiotics, or other antimicrobial agents, such as silver salts, nitrofurazone, chlorhexidine, polymerized quaternary ammonium surfactants, antibacterial peptides and anionic nanoporous hydrogels. ${ }^{3}$ These approaches are designed to kill bacteria through direct contact with the incorporated microbiocidal agents. However, these strategies have a number of drawbacks such as cost, the 
toxicity of the additives, a limited operational lifetime related to the depletion of the active antibacterial agents from the coating over time, and the potential to contribute toward the potential development of antibiotic resistant bacterial strains due to an inability to maintain a therapeutic dose throughout the lifetime of the device. Consequently, new strategies to combat biofouling have begun to focus on the design of new materials that exhibit an inherent resistance to bacterial attachment in order to minimize biofilm formation. ${ }^{4}$ In this case "inherent" defines that the microbial resistance is achieved from the molecular structure and/or ordering of the polymer alone, with no additive or active required. Furthermore, in the case of these copolymers, it has been shown that they do not kill the bacteria and so do not contribute to the potential selection of resistant strains. Such bacterial attachment-resistant (BAR) materials could be used on their own or in conjunction with traditional microbiocidal agent impregnation approaches to achieve greater efficacy of the coating. ${ }^{5}$ To date, few materials have successfully achieved this anti-attachment strategy to produce low-fouling coatings. Those that have been reported include poly(ethylene glycol) brushes, ${ }^{6}$ and zwitterionic polymers, ${ }^{7}$ which have achieved reductions in bacterial attachment of between 1.4 and 25 fold respectively when compared with a native glass surface.

Over the last few decades the use of high throughput materials discovery microarray screening methods to assess polymers has been demonstrated to be a powerful tool in identifying materials with desirable properties. ${ }^{8}$ For example, this type of microarray screening approach has previously proven useful for identifying polymeric materials that support stem cell self-renewal. ${ }^{8 c,}{ }^{8 f}$ Using such high throughput microarray methods and commercially available monomers, the authors reported the screening of more than 1300 unique copolymers in nearly 20,000 different bacterial attachment assays. ${ }^{9}$ This study led to the identification of the specific molecular motifs in the monomers, and their daughter 
polymers leading to the discovery of a new class of bacterial resistant synthetic polymers that produced the highest levels of BAR performance in this first generation screen. ${ }^{9}$

These resultant "hit" polymers (i.e. those exhibiting high BAR performance) were shown to exhibit inherent bacterial anti-attachment properties, in other words the polymers alone generated up to a 30-fold reduction in bacterial attachment compared to silver hydrogel catheters without the need for a bio-active additive. 9 "The BAR performance exhibited by these coating materials has been attributed to both their weakly amphiphilic nature and the molecular rigidity of the polymers' pendant groups. ${ }^{10}$ For example, the BAR resistance of a certain subset of these polymers correlated with the presence of pendant hydrophobic aromatic and cyclic aliphatic hydrocarbon groups only when accompanied by the ester moiety of a (meth)acrylate, which constituted the polymerizable center. The former provided both the pendent group rigidity and a segment of hydrophobic character whilst the latter provided a segment with some hydrophilic nature. Comparison of the BAR performance of these "hit" monomers with poly(styrene), a very hydrophobic material that contains aromatic pendant groups not associated with an ester group that is known to support bacterial attachment, indicated that the ester group was important in attachment prevention. If polymers that exhibit good BAR performance are to be successfully commercially exploited in coating applications, they also need to possess suitable mechanical and adhesion properties such that they can function as viable coatings for a specific target surface/bulk materials. For example, they may be applied as a coating upon a device such as a urinary catheter and thus will need to exhibit the same level of flexibility as the matrix material from which catheter has been made. This will ensure that the resulting coating neither fails in use (e.g. appearance of cracks) nor restricts the ability to insert the device. Similarly, it will have to adhere well to the surface structure of the material used to construct the device, so that the coating does not 
delaminate and expose regions of the native surface, which are likely to promote biofilm formation.

This paper details initial studies into the molecular optimization and scale up of these weakly amphiphilic coating polymeric materials as a first step toward developing commercially viable coating materials that exhibit good anti-attachment performance. The initial targets were to: (a) obtain "hit" copolymers that exhibit a glass transition temperature ( $\left.T_{g}\right)$ such that they would exhibit flexibility that matched that of the catheter material, but would not be prone to excessive creep at manufacturing/body temperatures (here this was defined as a $\mathrm{T}_{\mathrm{g}}$ in the range to -20 to $15^{\circ} \mathrm{C}$ ), (b) pass the surface adhesion/compatibility test set for the target catheter application, (c) increase hydrophilicity of the coating without the degradation of the bacterial attachment-resistance and (d) achieve successful scale up from a microarray spot to a $50 \mathrm{~g}$ batch scale.

\section{Experimental}

Materials; All materials were used as supplied unless stated otherwise. All monomeric species used in this study, ethylene glycol dicyclopentenyl ether acrylate (EGDPEA), benzyl acrylate (BA), benzyl methacrylate (BMA, 96\%), diethylene glycol ethyl ether methacrylate (DEGMA, 95\%), 3,3,5-trimethyl cyclohexyl methacrylate (TMCHMA, 98\%) and isobornyl methacrylate (IBMA), were purchased from Sigma Aldrich. Bis[(difluoroboryl) diphenylglyoximato]cobalt(II) (PhCoBF, DuPont) was used as supplied without further purification. 2,2'-Azobis(4-methoxy-2,4-dimethylvaleronitrile) (V70, 98\%, Sigma Aldrich) was purified by recrystallization with methanol. Dichloromethane (DCM) was molecularly distilled then dried with molecular sieves.

\section{Polymer synthesis - General Procedure for Catalytic Chain Transfer Polymerization (CCTP):}

The typical protocol used for the catalytic chain transfer polymerization synthesis of poly("'hit"'-co-DEGMA), where "hit" designates one of the monomers of interest identified 
from the high throughput screening assessment, was as follows. Bis[(difluoroboryl) diphenylglyoximato] cobalt(II) (PhCoBF, $500 \mathrm{ppm}$ for acrylate/5 ppm for methacrylate) and 2,2'-Azobis(4-methoxy-2,4-dimethyl valeronitrile) (0.5 mol\% relative to monomer) were added to a $500 \mathrm{~mL}$ flask under an inert atmosphere. The appropriate quantities of the "hit" monomers required to make the target (co)polymer, were then added to the flask in the necessary predefined molar ratio along with dichloromethane (DCM) which was the reaction solvent in a 1:3 v:v ratio. The subsequent reaction solution was then degassed using at least two freeze-pump-thaw cycles and an inert argon atmosphere introduced to the flask. The reaction mixture was then raised to and held at $35^{\circ} \mathrm{C}$ for $24 \mathrm{~h}$ during which time the polymerization occurred. After $24 \mathrm{~h}$, the polymerization was terminated by cooling the contents to room temperature and exposure of the reaction mixture to air with rapid stirring. NMR spectroscopic analysis was carried out on the crude polymerization solution to determine conversion and overall monomer composition. The DCM was removed under reduced pressure at room temperature and this crude polymer was dissolved in HPLC grade THF for GPC analysis, then the sample was precipitated into an excess of hexane and dried prior to for DSC analysis being conducted.

Polymer/material characterization - Gel Permeation Chromatography (GPC): In a typical GPC analysis, an HPLC pump was employed as the solvent delivery system to maintain a constant, pulseless rate of solvent flow at $1 \mathrm{~mL} \mathrm{~min}^{-1}$. The polymer solution in THF was prepared in a GPC vial with Teflon cap at concentration of approximately $7 \mathrm{mg} \mathrm{mL} \mathrm{m}^{-1}$. The sample was then injected to the GPC system by an autosampler. The solvent transports the sample through a series of three columns: one PLgel $5 \mu \mathrm{m}$ Guard column (Polymer Laboratories) and then two PLgel $5 \mu \mathrm{m}$ MIXED-C columns (Polymer Laboratories) which were kept at $35{ }^{\circ} \mathrm{C}$. The two PLgel $5 \mu \mathrm{m}$ MIXED-C columns were used to separate the polymer samples into weight fractions to determine the molecular weight (both number 
average $\left(M_{n}\right)$ \& weight average $\left.\left(M_{w}\right)\right)$ and dispersity $(Đ)$. After separation, the polymer fractions were analyzed by the IR detector.

Nuclear Magnetic Resonance (NMR) Spectroscopy: ${ }^{1} \mathrm{H}$ and ${ }^{13} \mathrm{C}$ NMR spectroscopic analyses were recorded at $25{ }^{\circ} \mathrm{C}$ using Bruker DPX-300 and AV-400 MHz spectrometers. The samples were dissolved in deuterated chloroform $\left(\mathrm{CDCl}_{3}\right)\left(1 \mathrm{mg} \mathrm{mL}^{-1}\right)$ to which chemical shifts are referenced (residual chloroform at $7.26 \mathrm{ppm}$ ). Chemical shifts were recorded in $\delta_{\mathrm{H}}$ and $\delta_{\mathrm{C}}$ (ppm). Analysis of the spectra was carried out using ACDLABS 12 software.

Differential scanning calorimetry (DSC): Glass transition temperatures $\left(\mathrm{T}_{\mathrm{g}}\right)$ of the product polymers was determined using a Thermal Analysis (TA) Q2000 Differential Scanning Calorimeter (DSC) under a nitrogen stream (flow rate $50 \mathrm{~mL} \mathrm{~min}{ }^{-1}$ ). Changes in heat flow were recorded between $-50{ }^{\circ} \mathrm{C}$ and $90{ }^{\circ} \mathrm{C}$ over 3 cycles. A scan rate of $10{ }^{\circ} \mathrm{C} / \mathrm{min}$ with a 10 minute isotherm at either end of the range was employed. The instrument was calibrated using indium metal standards supplied by TA Instruments and has a quoted calorimetric precision and reproducibility of $\pm 0.05 \%$ and temperature control accuracy of $\pm 0.1{ }^{\circ} \mathrm{C}$. Analysis of the data was performed using Universal Analysis by TA and a series of repeat measurements on a single polymer product showed the standard deviation from the mean $T_{g}$ was $\pm 0.35{ }^{\circ} \mathrm{C}$ using this software. Unless otherwise stated, the second scan was used to remove the thermal history of the polymer being analyzed.

Time of flight secondary ion mass spectrometry (ToF SIMS): ToF-SIMS measurements were conducted on an ION-ToF IV instrument operated using a monoisotopic $\mathrm{Bi}_{3}{ }^{+}$primary ion source operated at $25 \mathrm{kV}$ and in "bunched mode". A $1 \mathrm{pA}$ primary ion beam was used, and both positive and negative secondary ions were collected from a $100 \times 100 \mu \mathrm{m}$ area of each polymer coating over 10 -second acquisition time. Secondary ion masses were determined using a high resolution Time-of-Flight analyser allowing accurate mass assignment. The typical mass resolution (at m/z 41) was just over 6000 .

Page 7 of 24

ACS Paragon Plus Environment 
Material application testing: Dip coating and fatigue testing: Sectioned catheters were oxygen plasma treated for 5 minutes using a power of $50 \mathrm{~W}$ and initial oxygen pressure of 0.4 mbar. Treated catheters were immediately dip-coated with a $20 \%$ polymer solution in DCM for approximately 10 seconds before being gradually withdrawn from the solution. The samples were then dried in air overnight and at $<50$ mTorr for at least 7 days.| Polymer coatings were typically between 5 and 10 microns and an example SEM has been included in the ESI document as Figure S2. Fatigue testing was carried out on a Mayes Servohydraulic testing machine with a set of Instron wedge grips with ' $V$ ' jaws. The sample length between the two grips was 6 $\mathrm{cm}$. The sample was bent 500 times and the total distance of movement between the two grips during bending was $1 \mathrm{~cm}$.

Bacterial strains and assays: Three bacterial species, ${ }^{9}$ Pseudomonas aeruginosa strainPAO1, Staphylococcus aureus strain 8325-4 and uropathogenic Escherichia coli strain 536 were routinely grown on either LB (Luria-Bertani, Oxoid, UK) agar plates at $37{ }^{\circ} \mathrm{C}$ or in broth at $37{ }^{\circ} \mathrm{C}$ with $200 \mathrm{rpm}$ shaking. ${ }^{9}$ Bacterial cells were inoculated onto $4 \mathrm{~cm}$ sections of coated catheters as previously described. ${ }^{9}$ Briefly, prior to incubation with the bacteria, the coated catheters were washed in distilled $\mathrm{H}_{2} \mathrm{O}$ for $10 \mathrm{~min}$ and air-dried. UV-sterilized catheters were incubated in $10 \mathrm{~mL}$ RPMI-1640 defined medium (Sigma, UK) inoculated with bacteria $\left(\mathrm{OD}_{600}=0.01\right)$ from overnight cultures grown at $37{ }^{\circ} \mathrm{C}$ with $60 \mathrm{rpm}$ shaking. At the desired time points, the catheters were removed ( 0 to $72 \mathrm{~h}$ ), and washed three times with $15 \mathrm{~mL}$ phosphate buffered saline (PBS, Oxoid, UK) at room temperature for $5 \mathrm{~min}$. After rinsing with distilled $\mathrm{H}_{2} \mathrm{O}$ to remove salts, the catheters were stained with $20 \mu \mathrm{M}$ SYTO17 dye (Invitrogen, UK) at room temperature for $30 \mathrm{~min}$. After air drying, the samples were examined using a Carl Zeiss LSM 700 Laser Scanning Microscope with ZEN 2009 imaging software (Carl Zeiss, Germany). Bacterial surface coverage was quantified using open source Image J 1.44 software (National Institute of Health, US). For scanning electron microscopy 
(SEM), the washed samples were fixed, gold coated and examined using a Philips XL30 Scanning Electron Microscope. For cross-section images samples, were first frozen in liquid nitrogen before cutting with a razor blade.

\section{Results and Discussion}

In this study, a range of copolymers was synthesized using a library containing five acrylate/methacrylate monomers which had demonstrated good BAR performance in initial high throughput screening. ${ }^{9}$ A sixth monomer (diethylene glycol methyl ether methacrylate DEGMA), was included to allow the glass transition point $\left(\mathrm{T}_{\mathrm{g}}\right)$ and level of hydrophilicity of the final copolymer to be controlled to match key physical properties with that of the device that was to be coated and its intended application. The molecular structures of these monomers are shown in Figure 1.

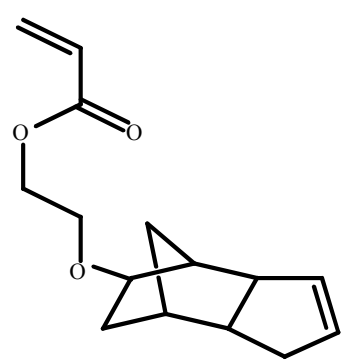

Ethylene glycol dicyclopentenyl ether acrylate (EGDPEA)

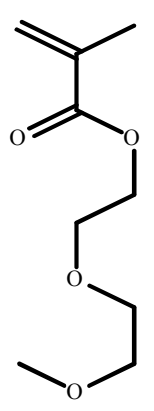

Diethylene glycol methyl ether methacrylate (DEGMA)

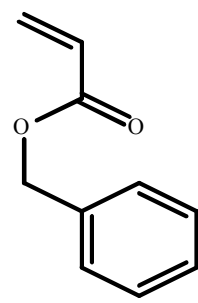

Benzyl acrylate

(BA)

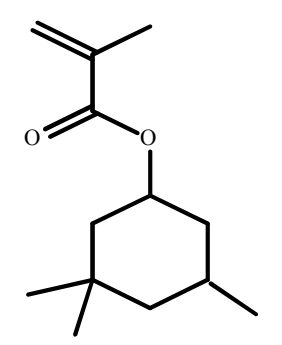

3,3,5-trimethylcyclohexyl methacrylate (TMCHMA)

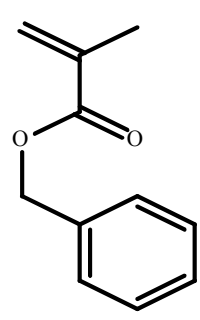

Benzyl methacrylate (BMA)

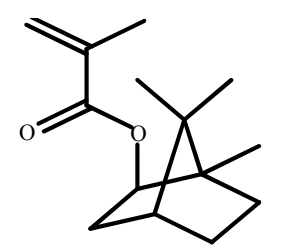

Isobornyl methacrylate (IBMA)

Figure 1. Structures of monomers used in the synthesis of the copolymers 
DEGMA homopolymer has previously been shown not to exhibit bacterial attachment resistance. ${ }^{9,11}$ The aim was to investigate the effect that introducing DEGMA had upon both BAR and material properties and so begin to define how to optimize the biofilm resistant coating copolymer material for use in medical devices.

Polymers were synthesized using catalytic chain transfer free radical polymerization (CCTP) methods to ensure control of molecular weight (Mwt) down to the oligomeric level if required. ${ }^{12}$ Furthermore, this polymerization technique was used because it does not leave any additional inorganic functional group on the polymer as a result of the control mechanism, which may affect the overall performance/toxicity of the material. ${ }^{12}$ For example while thiol chain transfer agents introduce a sulfur containing heat group, the CCTP mechanism leaves the polymer with only an unsaturated terminal group that is equivalent to that which results from disproportionation termination. Additionally, this pre-polymerization strategy also allowed assessment of any effects that the Mwt of the copolymer had on the dip coating process, final coating integrity and/or BAR performance. The anti-bacterial attachment performance was tested by culturing bacteria on polymer dip-coated silicone catheters where commercial catheters were used as benchmarking controls of performance.

Molecular Structure Optimization: The initial solution homopolymerization of ethylene glycol dicyclopentenyl ether acrylate (EGDPEA) using this strategy resulted in acrylate polymers that were brittle. This observation was linked to the $\mathrm{T}_{\mathrm{g}}$ of the homopolymer being too high for the target application, (see Table 1, Entry 1). To address this issue and achieve the desired target material property modification, copolymerization of several "hit" monomers with diethylene glycol methyl ether methacrylate (DEGMA) was conducted. DEGMA was chosen because its incorporation into the polymer will lower the $\mathrm{T}_{\mathrm{g}}$ of the copolymer improving its physical compatibility with the test catheter matrix material (silicone) and it hydrophilic nature should lead to the formation of hydrated coatings.

Page 10 of 24 
Table 1. Physical and Molecular Characteristics of 'Hit' Monomer Containing Copolymers Synthesized by Solution CCTP.

\begin{tabular}{|c|c|c|c|c|c|c|c|}
\hline & Monomer(s) & $\begin{array}{c}\text { Feed Ratio } \\
\mathbf{( \% )}\end{array}$ & $\begin{array}{c}\text { Copolymer } \\
\mathbf{R a t i o}^{\mathbf{a}} \\
\mathbf{( \% )}\end{array}$ & $\begin{array}{c}\mathbf{C o n v} \\
(\mathbf{\%})\end{array}$ & $\begin{array}{c}\mathbf{M}_{\mathbf{n}} \\
\left(\mathbf{g ~ m o l}^{\mathbf{1}} \mathbf{)}\right.\end{array}$ & $\begin{array}{c}\mathbf{T}_{\mathbf{g ( o b s}} \\
\left({ }^{\circ} \mathbf{C}\right)\end{array}$ \\
\hline 1 & EGDPEA & 100 & 100 & 34 & 21,000 & 2.38 & 19 \\
\hline 2 & DEGMA & 100 & 100 & 48 & 5,600 & 3.29 & -36 \\
\hline 3 & EGDPEA/DEGMA & $75 / 25$ & $76 / 24$ & 46 & 27,300 & 1.69 & 9 \\
\hline 4 & EGDPEA/DEGMA & $50 / 50$ & $45 / 55$ & 44 & 19,400 & 1.58 & -6 \\
\hline 5 & EGDPEA/DEGMA & $25 / 75$ & $27 / 73$ & 25 & 6,400 & 2.62 & -40 \\
\hline 6 & BA:DEGMA & $75 / 25$ & $68 / 32$ & 70 & 32,300 & 1.77 & -4 \\
\hline 7 & BA:DEGMA & $50 / 50$ & $40 / 60$ & 61 & 21,400 & 1.52 & -17 \\
\hline 8 & BA:DEGMA & $25 / 75$ & $18 / 82$ & 75 & 4,100 & 2.77 & -42 \\
\hline 9 & BMA:DEGMA & $75 / 25$ & $68 / 32$ & 29 & 28,600 & 1.38 & 22 \\
\hline 10 & BMA:DEGMA & $50 / 50$ & $42 / 58$ & 62 & 16,400 & 2.17 & 1 \\
\hline 11 & BMA:DEGMA & $25 / 75$ & $26 / 74$ & 19 & 4,000 & 2.56 & -27 \\
\hline 12 & IBMA:DEGMA & $75 / 25$ & $73 / 27$ & 52 & 19,400 & 1.62 & 74 \\
\hline 13 & IBMA:DEGMA & $50 / 50$ & $48 / 52$ & 53 & 13,900 & 1.88 & 25 \\
\hline 14 & IBMA:DEGMA & $25 / 75$ & $25 / 75$ & 89 & 2,900 & 1.69 & -10 \\
\hline 15 & TMCHMA:DEGMA & $75 / 25$ & $71 / 29$ & 61 & 19,500 & 1.6 & 50 \\
\hline 16 & TMCHMA:DEGMA & $50 / 50$ & $47 / 53$ & 48 & 13,600 & 1.68 & 24 \\
\hline 17 & TMCHMA:DEGMA & $25 / 75$ & $23 / 77$ & 54 & 9,000 & 1.78 & -12 \\
\hline
\end{tabular}

a) Defined by NMR analysis. An example NMR has been included as Figure S1 in the extra scientific information document and example integrals calculation as Table S1.

As well as $T_{g}$, the level of chain entanglement can also affect the level brittleness exhibited by the copolymer. Therefore, the introduction of DEGMA which has an "oligomeric" pendant group into the copolymer also affects the level of chain interaction and hence entanglement within these new copolymers. Thus, addition of DEGMA will tend to reduce both of these chain characteristics by disrupting the chain packing and so lead to a further reduction in the likelihood of the material exhibiting brittle failure. The copolymers synthesized within this study are detailed in Table 1.

Experiments were conducted to; (a) define the optimum copolymerization ratios required to deliver a decrease in the $T_{g}$ of materials containing the differing "hit" monomers to improve material flexibility such that coatings can be developed with material properties that match those of the silicone catheter matrix, (b) investigate how the inclusion of DEGMA at varying levels affected the onset of gelation during the reaction and (c) assess whether 
copolymers with these optimum ratios retained sufficient bacterial attachment resistance to be candidate BAR coating materials.

To investigate the ability to tune the $\mathrm{T}_{\mathrm{g}}$ of the resultant copolymer, three representative feed ratios ("hit":DEGMA ratio $=75 / 25,50 / 50$, and 25/75) were used and the resulting copolymers were subjected to both spectroscopic analysis (to define composition) and bacterial attachment resistance assays to confirm fitness for purpose. NMR spectroscopic analysis of the copolymers showed that the final compositions were typically close to the target levels (Table 1). These results defined that the reactivity ratios of the monomer used were sufficiently alike that the polymerization process was robust enough for commercial manufacture of the target coating materials with an acceptable level of batch to batch reproducibility. The $\mathrm{T}_{\mathrm{g}} \mathrm{s}$ of the resultant copolymers were then measured experimentally and plotted against the values predicted by the Fox equation (see Figure 2) and were found to be in good agreement showing a linear trend $\left(\mathrm{R}^{2}=0.95\right) .{ }^{13}$ Deviation from the predicted value is likely due to residual high boiling monomer within the copolymer material.

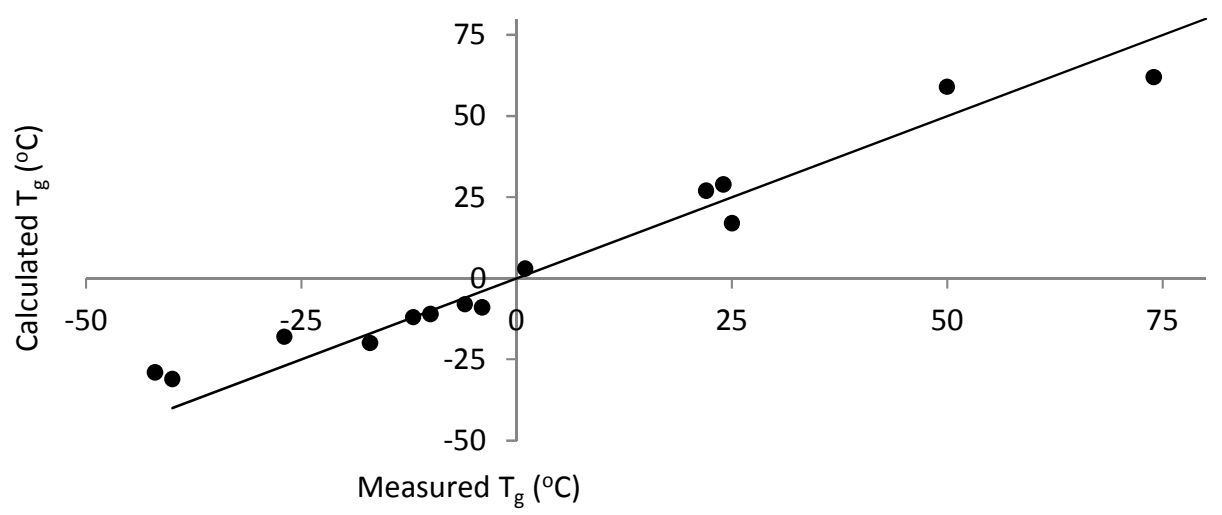

Figure 2. Comparison of the observed $\mathrm{T}_{\mathrm{g}} \mathrm{S}$ with those calculated by the Fox equation. ${ }^{13}$ The $\mathrm{y}=\mathrm{x}$ line is drawn, $\mathrm{R}^{2}=0.95$.

The linear trend in the data confirmed that this polymerization technique could be used to predictably deliver a copolymer with a target $\mathrm{T}_{\mathrm{g}}$ by measuring that of the "hit" homopolymers and that these systems are robust enough to allow the fine tuning of the thermal and 
potentially the consequent mechanical properties. Subsequent experiments investigated the material/bacterial attachment properties of the polymer initially using the lowest level of DEGMA incorporation. The data in Table 1 show that of the $75: 25 \mathrm{~mol} \%$ ratio polymers synthesized only EGDPEA/DEGMA and BA:DEGMA exhibited a $\mathrm{T}_{\mathrm{g}}$ that was below room temperature and thus likely to produce a flexible coating. Thus these combinations were selected for further study.

The molecular weights of the resulting polymers were shown to typically reduce as the DEGMA concentration increased. Since the copolymerizations had been conducted using CCTP in DCM at $35^{\circ} \mathrm{C}$, this was attributed to two effects. Firstly, in the case of the acrylate "hit" monomers, CCTP catalysts cannot control the polymerization of acrylates as efficiently as methacrylates. This is because of the propensity to form a more stable $\mathrm{Co}-\mathrm{C}$ bond between $\mathrm{CoPhBF}$ and the radical species of the growing chain, which slows the reaction rate. ${ }^{12}$ Therefore, increasing the methacrylate content of the monomer mixture by increasing DEGMA concentration resulted in more efficient CCTP chain transfer and so a lower overall molecular weight. In the case of the methacrylate "hit" monomers, the increase in DEGMA concentration led to a reduction in the system viscosity because the reaction temperature employed is $\sim 70{ }^{\circ} \mathrm{C}$ above DEGMA's $\mathrm{T}_{\mathrm{g}}$ but is closer to or below that of the "hit" methacrylates. Thus any diffusion limitations that CCTP catalysts encounter are reduced as the lower $\mathrm{T}_{\mathrm{g}}$ monomer concentration is increased, and therefore they operate more efficiently resulting in a lower molecular weight product. The 75:25 mol\% ratios were again highlighted as the candidates to be taken forward because they produce the higher molecular weights and so were likely to make the most viable coatings. Nevertheless, the effect of varying DEGMA concentration on the Mwts of the final polymers requires further investigation to confirm these proposed hypotheses.

Page 13 of 24 
It was also noted that the $Đ$ values of the copolymers typically increase with increasing DEGMA concentration. This was attributed to a combination of the "oligomeric" nature and hydrophilic character of the EO chain forming the pendant group in this monomer. Therefore, this group will influence both its relative reactivity compared to the other monomers due to its steric bulk/ pendant chain flexibility and the GPC analysis of the homo/copolymers, where it will influence the hydrodynamic volume when compared with the hydrophobic standards containing smaller, non-oligomeric pendant groups. The influence of these combined effects are reflected in the experimentally recorded $Đ$ for the DEGMA homopolymer of $>3$.

This conclusion was also supported by the fact that the 50/50 copolymers with the acrylate monomers, i.e. EGDPEA/DEGMA and BA:DEGMA, did not appear to follow this trend. This observation was considered to be due to the reduced steric bulk around the polymerizable double bond of the acrylates, which allowed easier initial copolymerization, so resulting in a lower $Đ$ than the methacrylate equivalents. $Đ$ was then noted to increase as the DEGMA content in the polymer was raised to $75 \%$ so that its properties dominate.

The 25/75 copolymer of these acrylate monomers also exhibited $\mathrm{T}_{\mathrm{g}} \mathrm{s}$ below that recorded by the experimentally measured value of the DEGMA homopolymer. However, this was within experimental error at these low temperatures, which is reflected in the broad range of literature-quoted values for DEGMA homopolymer. Hence it was necessary to determine the value experimentally in this study.

The onset of gelation during the polymerization was investigated with the DEGMA included copolymers. The initial copolymerization work employed a 50:50 v/v comonomer:solvent ratio. Thus, a series of polymerizations were conducted in which this relative comonomer:solvent ratio was varied to investigate the effect that increasing the monomer/polymer concentration in the reaction medium has upon both the onset of gelation and the rate of conversion achieved. The results of these experiments, which used a 75:25

Page 14 of 24 
ratio EGDPEA:DEGMA relative mol\% monomer feed concentration as a model system, are shown in Table 2.

Table 2 - Effect of solvent concentration upon the copolymerization of EGDPEA:DEGMA with a relative monomer molar feed concentration of $75 \%: 25 \%$ conducted using CCTP

\begin{tabular}{|c|c|c|c|c|c|c|c|c|}
\hline Entry & $\begin{array}{c}\text { Monomer } \\
/ \\
\text { DCM } \\
\% \text { ratio }\end{array}$ & $\begin{array}{c}\text { Temp } \\
\left({ }^{\circ} \mathrm{C}\right)\end{array}$ & $\begin{array}{c}\text { Time } \\
(\mathrm{h})\end{array}$ & $\begin{array}{c}\text { EGDPEA } \\
\text { Conv } \\
(\%)\end{array}$ & $\begin{array}{c}\text { DEGMA } \\
\text { Conv } \\
(\%)\end{array}$ & $\begin{array}{c}\text { Overall } \\
\text { Conv } \\
(\%)\end{array}$ & $\begin{array}{c}\mathrm{M}_{\mathrm{n}} \\
\left(\mathrm{g} \mathrm{mol}^{-1}\right)\end{array}$ & Đ \\
\hline 1 & $100: 0$ & 40 & 1 & \multicolumn{6}{|c|}{ Gelation } \\
\hline 2 & $100: 0$ & 35 & 3 & \multicolumn{6}{|c|}{ Gelation } \\
\hline 3 & $25: 75$ & 35 & 24 & 45 & 47 & 46 & 27500 & 1.69 \\
\hline 4 & $75: 25$ & 35 & 24 & 44 & 66 & 48 & 58500 & 3.63 \\
\hline 5 & $50: 50$ & 35 & 24 & 47 & 58 & 50 & 35000 & 2.52 \\
\hline 6 & $25: 75$ & 35 & 48 & 68 & 94 & 75 & 41000 & 2.96 \\
\hline
\end{tabular}

The bulk copolymerization (i.e. polymerization conducted in the absence of solvent) led to gelation after $1 \mathrm{~h}$ and $3 \mathrm{~h}$ at $40^{\circ} \mathrm{C}$ and $35^{\circ} \mathrm{C}$, respectively (Table 2, Entries 1 and 2) suggesting that the use of solvent to dilute the monomer concentration was essential. Consequently, all subsequent copolymerizations were conducted at $35{ }^{\circ} \mathrm{C}$ to reduce the risk of reaching the gel point prior to terminating the polymerization. Three different total monomer:DCM volume ratio of 25\%:75\%, 50\%:50\% and 75\%:25\% v:v were then examined (Table 2, Entries 3-5) to define the influence of solvent concentration on the polymerization. Adopting this reaction temperature resulted in an initiator half-life of approximately $10 \mathrm{~h}$, thus as the $24 \mathrm{~h}$ polymerization time represents only 2 half-life turnovers, it was found to deliver an overall $\sim 50 \%$ yield as would be expected. Reducing the volume of DCM did not significantly affect the overall conversion achieved, but both the $M_{n}$ and the $Đ$ values were noted to increase, implying that the formation of branched polymers was increasingly likely to occur at lower solvent concentrations. Additionally, the relative level of DEGMA incorporation increased as the solvent concentration was reduced. This was attributed to the increased likelihood of DEGMA being incorporated into the chain because the reaction temperature employed was $\sim 70{ }^{\circ} \mathrm{C}$ higher than DEGMA's $\mathrm{T}_{\mathrm{g}}$, but only $\sim 15{ }^{\circ} \mathrm{C}$ higher than 
that of EGDPEA. Thus, relative to EGDPEA, the physical form of DEGMA will be more "fluid-like", and so it is much less likely to encounter diffusion limitations and thus reactivity issues as the reaction media become more viscous as the solvent concentration is reduced. It was concluded from the data in Table 2 that, for a 75 mol\% EGDPEA: 25 mol\% DEGMA copolymer, then a $25 \%: 75 \% \mathrm{v}: \mathrm{v}$ comonomer:solvent ratio exhibits the best conditions for achieving equivalent co-monomer reactivity ratios.

Consequently, a reaction was conducted using a 25:75 v:v monomer:DCM for $48 \mathrm{~h}$ at $35^{\circ} \mathrm{C}$ (Table 2, Entry 6), in an attempt to obtain higher overall conversion. However, whilst increasing the time to 4 half-life turnovers did deliver the desired increase in conversion in the $48 \mathrm{~h}$ reaction, it was associated with an increase in $\mathrm{M}_{\mathrm{n}}$ and the $Đ$ indicating the onset of branching within the system. Since these increases were in relation to a rise in DEGMA incorporation, this suggested that this was as a result of higher viscosity of the reaction medium as conversion increased.

Therefore, due to the observed issues that are related to the viscosity-related onset of gelation, it was concluded that the strategy for bacterial assessment was to synthesize these polymers in the form of a polymer in solvent/monomer solution (or resin) for direct dip coating the test catheters. The polymerization conditions chosen to give the best opportunity to achieve equivalent co-monomer and prevent the onset of branching in these dip coating resins was a volume ratio of monomer:DCM of $25 \%: 75 \% \mathrm{v}: \mathrm{v}$ with a reaction time $24 \mathrm{~h}$ at 35 ${ }^{\circ} \mathrm{C}$. Coated samples were characterized by SEM prior to use in bacterial assays (see Figure S2 in the ESI document).

Bacterial Attachment Assessment: To quantify the effect that the addition of DEGMA into "hit" materials had upon bacterial attachment, the surface coverage of three different bacterial species upon the surface of the copolymers detailed in Table 1 was quantified. Both the

Page 16 of 24 
results for the each species and the average surface coverage across three different species are shown in Figure 3. Commercial silicone and silver hydrogel catheters were used to benchmark the performance of the "hit" materials.

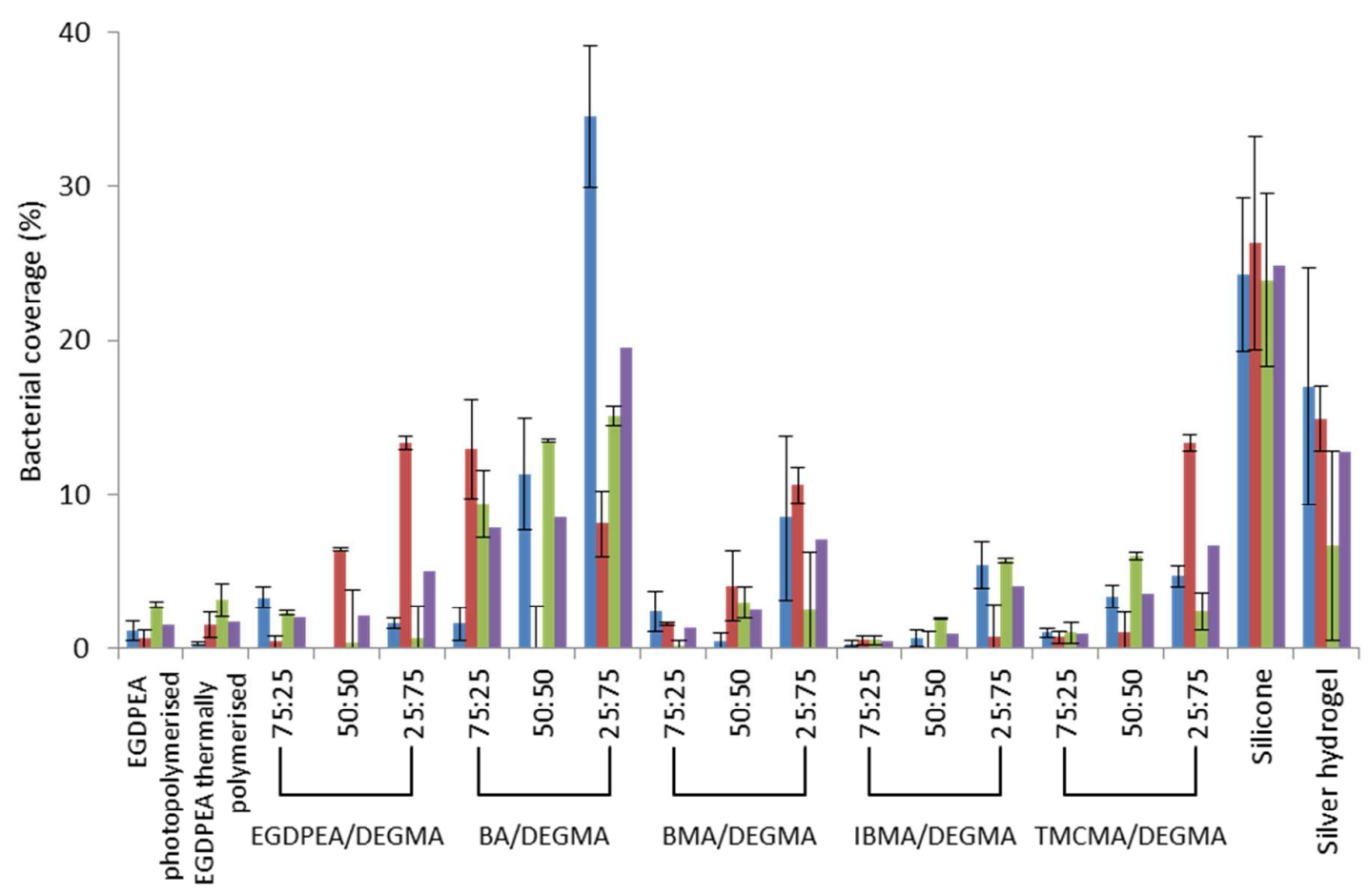

Figure 3. Bacterial coverage of three different strains after $72 \mathrm{~h}$ culture where $\mathrm{PA}=P$. aeruginosa, PAO1 (blue/first bar), $\mathrm{SA}=S$. aureus $8325-4$, (red/second bar), UPEC = uropathogenic E. coli 536 (green/third bar). Average bacterial attachment across the three different strains (purple/fourth bar). No homopolymer DEGMA data is presented in this figure because this material did not form a viable stable film for subsequent material testing under the experimental conditions used. DEGMA materials only proved sufficiently stable when copolymerized.

The data in Figure 3 shows that the catheters coated with the copolymers containing "hit" monomers showed reduced bacterial attachment compared with the silicone catheters and, except for the 25:75 BA/DEGMA polymer, attracted less than the commercially available silver hydrogel coated catheter. The polymer with the overall lowest bacterial attachment was the 75:25 IBMA/DEGMA polymer with a bacterial coverage less than $0.5 \%$ for all three bacterial species. For comparison a homopolymer of EGDPEA was also synthesized by photoinitiated polymerization similar to the in-situ polymerization method used for polymer 
microarray synthesis. The biological performance of the photo- and thermal- initiated EGDPEA homopolymers were identical within the error of the measurement, indicating that both synthetic routes are viable for producing coatings for catheters. For all copolymers, the average bacterial attachment increased with increasing DEGMA concentrations, suggesting that DEGMA compromises the anti-attachment performance as would be expected as we are "diluting" the level of "hit" monomer in the copolymer structure. However, even with less 50 $\%$ of the "hit" monomer all the copolymer-coated catheters outperformed the commercially available silver hydrogel coated device. Furthermore, the level of variation in molecular weight and dispersity exhibited by the "hit":DEGMA copolymers did not appear to adversely influence their bacterial attachment resistance, rather it appeared to be dominated by the DEGMA content. This was primarily attributed to the substitution of a lower performing monomer but may also be chain length related as the inclusion of DEGMA generally resulted in a significant reduction in Mwt. At present our understanding of the influence that the acrylate and methacrylate fragment has upon BAR performance not been fully defined and is currently under investigation. Additionally, polymer coatings were typically between 5 and 10 microns and an SEM of a typical coating has been included as Figure S2 in the ESI document, In this range we have not observed any influence of coating thickness on resistance to bacterial attachment.

Material Property Optimization - Surface composition: Understanding the chemical composition of the surface is critical in interpreting bacterial attachment as bacteria only contact the top surface of materials they attach to. Time of flight secondary ion mass spectrometry (ToF SIMS) was used to qualitatively characterize the level of DEGMA presence on the surface, and the result is shown in Figure 4.

Page 18 of 24 


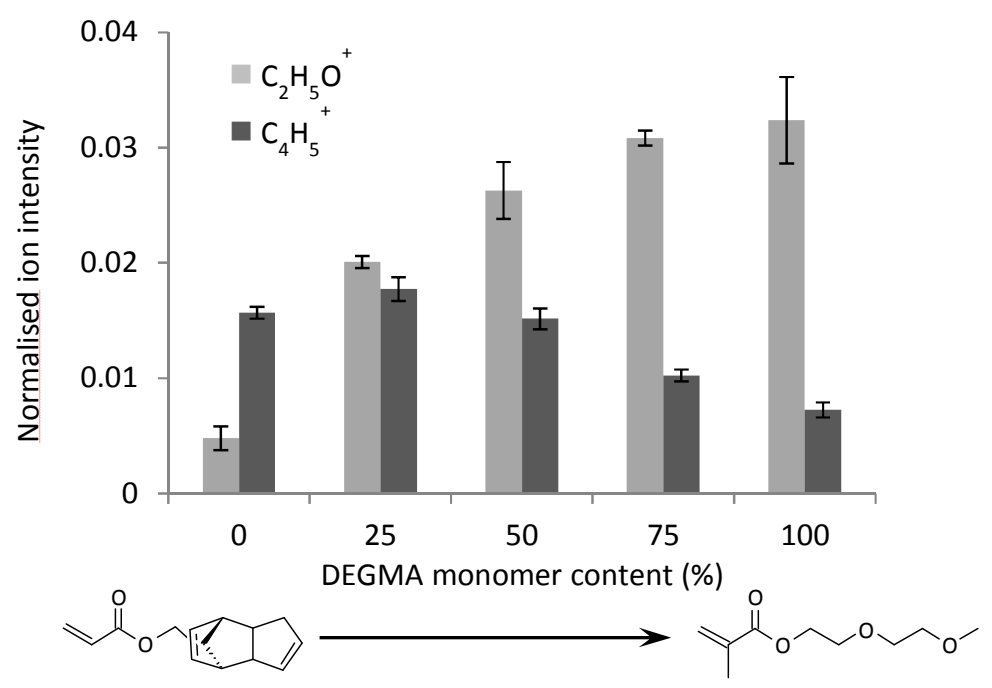

Figure 4. The level of DEGMA present on the surface of the EGDPEA/DEGMA copolymers. Secondary ion $\mathrm{C}_{2} \mathrm{H}_{5} \mathrm{O}^{+}$was chosen as an indicative DEGMA daughter ion, whilst secondary ion $\mathrm{C}_{4} \mathrm{H}_{5}{ }^{+}$was chosen as an indicative EGDPEA daughter ion.

The data in Figure 4 show that the intensity of the $\mathrm{C}_{2} \mathrm{H}_{5} \mathrm{O}^{+}$species, which is the indicative ion of for DEGMA, increased with the increasing amount of DEGMA in the copolymer. Meanwhile, that of $\mathrm{C}_{4} \mathrm{H}_{5}^{+}$, which is a characteristic secondary ion of EGDPEA, decreased with decreasing concentration of EGDPEA. This indicates that the addition of DEGMA in bulk also resulted in the increase of its concentration on the surface. The ion images for characteristic ions showed no evidence of micron-scale phase separation on the polymer. Material Property Optimization - Fatigue Testing: To determine whether the copolymers are sufficiently flexible as a layer of thin coating on the silicone catheter substrate, catheter segments were dip-coated in polymer resin solutions, dried and subjected to fatigue testing. The compressed side of the coating was examined by SEM to determine the integrity of the coating after repeated bending. SEM images of the compressed side of polymer coated silicone catheters after cyclic bending are shown in Figure 5. 

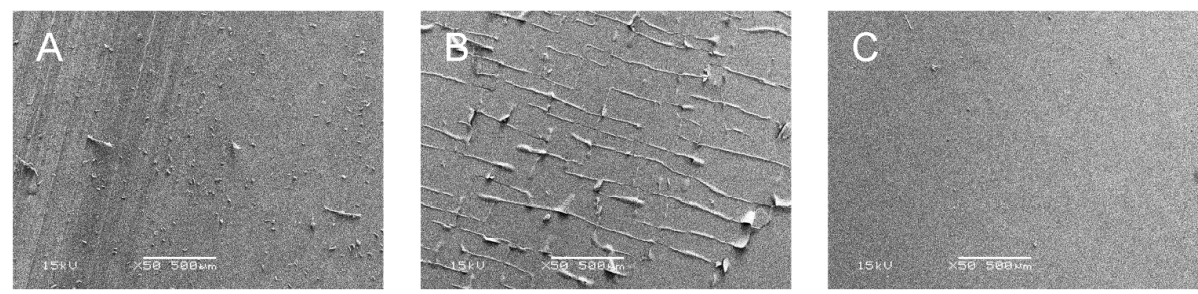

Figure 5. SEM images of polymer-coated silicone catheters after fatigue tests. Polymers were dip coated onto silicone catheters. (A) silicone without coating; (B) EGDPEA; (C)

EGDPEA/DEGMA(75/25)

Homopolymer EGDPEA was observed to show signs of wrinkling on the surface after the fatigue test, whilst the EGDPEA:DEGMA(75:25) copolymers showed no sign of coating damage. The $\mathrm{T}_{\mathrm{g}}$ of this copolymer is $9{ }^{\circ} \mathrm{C}$, which is below the testing temperature of $20{ }^{\circ} \mathrm{C}$, whilst that of homopolymer EGDPEA exhibits a $\mathrm{T}_{\mathrm{g}}$ of $19{ }^{\circ} \mathrm{C}$. This demonstrates the importance of lowering the Tg of "hit" copolymers to introduce flexibility to the copolymers. However, the potential influence of chain entanglement on these coating performance results also needs to be defined. Thus future work will report studies on the effects of molecular weight on the polymer with respect to bacterial attachment resistance and the level of brittleness exhibited, where the effect of entanglement will be investigated further by progressing to very low molecular weight.

\section{Conclusion}

Five "hit" monomers, which have been shown to produce homopolymers with significant antibacterial attachment resistance via high throughput polymer microarray screening and photo-induced polymerization, were successfully co-polymerized with DEGMA to lower the $\mathrm{T}_{\mathrm{g}}$ and so introduce increased flexibility using thermally induced polymerization. The aim of this study was to optimize the materials properties for application as coatings upon flexible silicone-based urinary catheters. It was demonstrated that the $\mathrm{T}_{\mathrm{g}}$ of resultant copolymers could be accurately estimated via the Fox equation, which means the $\mathrm{T}_{\mathrm{g}}$ can be further finetuned to meet other applications. Furthermore, the onset of gelation in the thermal polymerization was found to be dependent on the solvent concentration and reaction time. 
Optimum conditions to obtain the target copolymers were defined as a $25: 75 \mathrm{v}: \mathrm{v} \%$ monomer:solvent ratio at $35{ }^{\circ} \mathrm{C}$ for $24 \mathrm{~h}$. Increasing the DEGMA concentration in the reaction mixture lead to reduced molecular weight and to increased deviation from the target comonomer ratio in the final product. Both of these effects were attributed to a reduction in the viscosity in the reaction medium caused by increasing the DEGMA concentration that helps to overcome diffusion issues. The overall conclusion from the molecular optimization was that 75:25 mol\% EGDPEA:DEGMA copolymers were the key structures to review in the rest of the study. The bacterial attachment assays showed that although copolymerizations with DEGMA increased the bacterial copolymer surface coverage, it was still significantly lower on these copolymers compared with the benchmarks of silicone and silver hydrogel coated catheters, where the results for the $75: 25 \mathrm{v}: \mathrm{v} \%$ materials exhibited up to 25 -fold greater bacterial attachment. Additionally, these copolymer exhibited superior BAR to the benchmarks when up to $75 \%$ of the monomer mixture was DEGMA, with the exception of the BA:DEGMA where only up to $50 \%$ of the monomer feed could be DEGMA to achieve this. Furthermore, it was demonstrated that neither the variation in molecular weight / dispersity, nor the mode of initiating polymerizations (i.e. photo or thermal) affected the bacterial attachment resistance performance exhibited by the copolymers. ToF-SIMS analysis showed that the surface composition of the coating reflected the bulk composition of the polymer, and fatigue testing demonstrated that a 75:25 mol\% EGDPEA:DEGMA copolymer performed well as a functional coating as it was capable of being repeatedly flexed without delamination developing stress cracking unlike the homopolymer EGDPEA which failed this fatigue test.

Page 21 of 24

ACS Paragon Plus Environment 


\section{Supporting Information:}

This document contains two figures (a) an example ${ }^{1} \mathrm{H}$ NMR spectrum of precipitated IBMA:DEGMA 75/25 (300 $\left.\mathrm{MHz}, \mathrm{CDCl}_{3}\right)$ product to demonstrate how the copolymer ratios were defined and (b) an example SEM image of a cross-section of a coated catheter demonstrating that the coating is typically between 5-10 microns in thickness.

\section{Acknowledgements}

The authors would like to acknowledge the Impact Accelerators Account at the University of Nottingham for funding (Alexandra Gower) and Camstent Ltd for funding (Amy Stimpson). The Wellcome Trust (ref 085245 and 103882) are kindly acknowledged for funding this work. Morgan R. Alexander gratefully acknowledges the Royal Society for the provision of his Wolfson Research Merit Award.

\section{References}

1. (a) Banerjee, I.; Pangule, R. C.; Kane, R. S., Antifouling Coatings: Recent Developments in the Design of Surfaces That Prevent Fouling by Proteins, Bacteria, and Marine Organisms. Adv. Mater. 2011, 23 (6), 690-718; (b) Costerton, J. W.; Stewart, P. S.; Greenberg, E. P., Bacterial biofilms: A common cause of persistent infections. Science 1999, 284 (5418), 1318-1322.

2. Smith, A. W., Biofilms and antibiotic therapy: Is there a role for combating bacterial resistance by the use of novel drug delivery systems? Adv. Drug Delivery Rev. 2005, 57 (10), 1539-1550.

3. (a) Caillier, L.; de Givenchy, E. T.; Levy, R.; Vandenberghe, Y.; Geribaldi, S.; Guittard, F., Synthesis and antimicrobial properties of polymerizable quaternary ammoniums. Eur. J. Med. Chem. 2009, 44 (8), 3201-3208; (b) Costa, F.; Carvalho, I. F.; Montelaro, R. C.; Gomes, P.; Martins, M. C. L., Covalent immobilization of antimicrobial peptides (AMPs) onto biomaterial surfaces. Acta Biomater. 2011, 7 (4), 1431-1440; (c) Darouiche, R. O.; Mansouri, M. D.; Gawande, P. V.; Madhyastha, S., Efficacy of combination of chlorhexidine and protamine sulphate against device-associated pathogens. J. Antimicrob. Chemother. 2008, 61 (3), 651-657; (d) Greenfeld, J. I.; Sampath, L.; Popilskis, S. J.; Brunnert, S. R.; Stylianos, S.; Modak, S., Decreased bacterial adherence and biofilm formation on chlorhexidine and silver sulfadiazineimpregnated cnetral venous catheters implanted in swine. Crit. Care Med. 1995, 23 (5), 894-900; (e) Guay, D. R., An update on the role of nitrofurans in the management of urinary tract infections. Drugs 2001, 61 (3), 353-364; (f) Jaeger, K.; Osthaus, A.; Heine, J.; Ruschulte, H.; Kuhlmann, C.; Weissbrodt, H.; Ganser, A.; Karthaus, M., Efficacy of a benzalkonium chlorideimpregnated central venous catheter to prevent catheter-associated infection in cancer patients. Chemotherapy 2001, 47 (1), 50-55; (g) Li, P.; Poon, Y. F.; Li, W. F.; Zhu, H. Y.; Yeap, S. H.; Cao, Y.; Qi, X. B.; Zhou, C. C.; Lamrani, M.; Beuerman, R. W.; Kang, E. T.; Mu, Y. G.; Li, C. M.; Chang, M. W.; Leong, S. S. J.; Chan-Park, M. B., A polycationic antimicrobial and biocompatible hydrogel with microbe membrane suctioning ability. Nat. Mater. 2011, 10 (2), 149-156; (h) Yorganci, K.; Krepel, C.; Weigelt, J. A.; Edmiston, C. E., Activity of antibacterial

Page 22 of 24 
impregnated central venous catheters against Klebsiella pneumoniae. Intensive Care Med. 2002, $28(4), 438-442$.

4. Monds, R. D.; O'Toole, G. A., The developmental model of microbial biofilms: ten years of a paradigm up for review. Trends in Microbiol. 2009, 17 (2), 73-87.

5. Cloutier, M.; Mantovani, D.; Rosei, F., Antibacterial Coatings: Challenges, Perspectives, and Opportunities. Trends in Biotechnol. 2015, 33 (11), 637-652.

6. Holmes, P. F.; Currie, E. P. K.; Thies, J. C.; van der Mei, H. C.; Busscher, H. J.; Norde, W., Surface-modified nanoparticles as a new, versatile, and mechanically robust nonadhesive coating: Suppression of protein adsorption and bacterial adhesion. J. Biomed. Mater. Res., Part A 2009, $91 A(3), 824-833$.

7. (a) Cheng, G.; Li, G. Z.; Xue, H.; Chen, S. F.; Bryers, J. D.; Jiang, S. Y., Zwitterionic carboxybetaine polymer surfaces and their resistance to long-term biofilm formation. Biomaterials 2009, 30 (28), 5234-5240; (b) Cheng, G.; Zhang, Z.; Chen, S. F.; Bryers, J. D.; Jiang, S. Y., Inhibition of bacterial adhesion and biofilm formation on zwitterionic surfaces. Biomaterials 2007, 28 (29), 4192-4199.

8. (a) Anderson, D. G.; Levenberg, S.; Langer, R., Nanoliter-scale synthesis of arrayed biomaterials and application to human embryonic stem cells. Nat. Biotechnol. 2004, 22 (7), 863-866; (b) Celiz, A. D.; Harrington, H. C.; Hook, A. L., High throughput assessment and chemometric analysis of the interaction of epithelial and fibroblast cells with a polymer library. Appl. Surf. Sci. 2014, 313, 926-935; (c) Celiz, A. D.; Smith, J. G. W.; Patel, A. K.; Hook, A. L.; Rajamohan, D.; George, V. T.; Flatt, L.; Patel, M. J.; Epa, V. C.; Singh, T.; Langer, R.; Anderson, D. G.; Allen, N. D.; Hay, D. C.; Winkler, D. A.; Barrett, D. A.; Davies, M. C.; Young, L. E.; Denning, C.; Alexander, M. R., Discovery of a novel polymer for human pluripotent stem cell expansion and multilineage differentiation. Adv. Mater. 2015, 27 (27), 4006-4012; (d) Hook, A. L.; Anderson, D. G.; Langer, R.; Williams, P.; Davies, M. C.; Alexander, M. R., High throughput methods applied in biomaterial development and discovery. Biomaterials 2010, 31 (2), 187-198; (e) Tourniaire, G.; Collins, J.; Campbell, S.; Mizomoto, H.; Ogawa, S.; Thaburet, J. F.; Bradley, M., Polymer microarrays for cellular adhesion. Chem. Commun. 2006, (20), 2118-2120; (f) Zhang, R.; Mjoseng, H. K.; Hoeve, M. A.; Bauer, N. G.; Pells, S.; Besseling, R.; Velugotla, S.; Tourniaire, G.; Kishen, R. E. B.; Tsenkina, Y.; Armit, C.; Duffy, C. R. E.; Helfen, M.; Edenhofer, F.; de Sousa, P. A.; Bradley, M., A thermoresponsive and chemically defined hydrogel for longterm culture of human embryonic stem cells. Nat. Commun. 2013, 4, 1335.

9. Hook, A. L.; Chang, C.; Yang, J.; Luckett, J.; Cockrayne, A.; Atkinson, S.; Mei, Y.; Bayston, R.; Irvine, D. J.; Langer, R.; Anderson, D. G.; Williams, P.; Davies, M. C.; Alexander, M. R., Combinatorial discovery of polymers resistant to bacterial attachment. Nat. Biotechnol. 2012, 30 (9), 868-875.

10. Sanni, O.; Chang, C.; Anderson, D. G.; Langer, R.; Davies, M. C.; Williams, P. M.; Williams, P.; Alexander, M. R.; Hook, A. L., Bacterial attachment to polymeric materials correlates with molecular flexibility and hydrophilicity. Adv. Healthcare Mater. 2015, 4 (5), 695-701.

11. Hook, A. L.; Chang, C.; Yang, J.; Atkinson, S.; Langer, R.; Anderson, D. G.; Davies, M. C.; Williams, P.; Alexander, M. R., Discovery of novel materials with broad resistance to bacterial attachment using combinatorial polymer microarrays. Adv. Mater. 2013, 25 (18), 2542-2547.

12. (a) Gridnev, A. A.; Ittel, S. D., Catalytic chain transfer in free-radical polymerizations. Chem. Rev. 2001, 101 (12), 3611-3659; (b) Wang, W. X.; Stenson, P. A.; Marin-Becerra, A.; McMaster, J.; Schroder, M.; Irvine, D. J.; Freeman, D.; Howdle, S. M., New thiolate-cobalt(II) complexes for catalytic chain transfer polymerization of methyl methacrylate. Macromolecules 2004, 37 (18), 6667-6669.

13. (a) Cypcar, C. C.; Camelio, P.; Lazzeri, V.; Mathias, L. J.; Waegell, B., Prediction of the glass transition temperature of multicyclic and bulky substituted acrylate and methacrylate polymers using the energy, volume, mass (EVM) QSPR model. Macromolecules 1996, 29 (27), 8954-8959; (b) Fox, T. G., Influence of diluent and of copolymer composition on the glass transition temperature of a polymer system. Bull. Am. Phys. Soc. 1956, 1, 123; (c) Krause, S.; Gormley, J. J.; Roman, N.; Shetter, J. A.; Watanabe, W. H., Glass temperatures of some acrylic polymers. J. Polym. Sci., Part A:-Gen. Pap. 1965, 3 (10PA), 3573-3586; (d) Lu, Y. H.; Hsu, C. S.; Chou, C. H.; Chou, T. H., Preparation of poly(2,5-thienyleneethylene) and poly(2,5-furyleneethylene) by

Page 23 of 24 
vapor phase pyrolysis of (5-methyl-2-thienyl)methyl benzoate and (5-methyl-2-furyl)methyl benzoate. Macromolecules 1996, 29 (17), 5546-5550; (e) Shetter, J. A., Effect of stereoregularity on glass temperatures of a series of polyacryaltes and polymethacrylates. J. Polym. Sci., Part B: Polym. Lett. 1963, 1 (5), 209-213. 


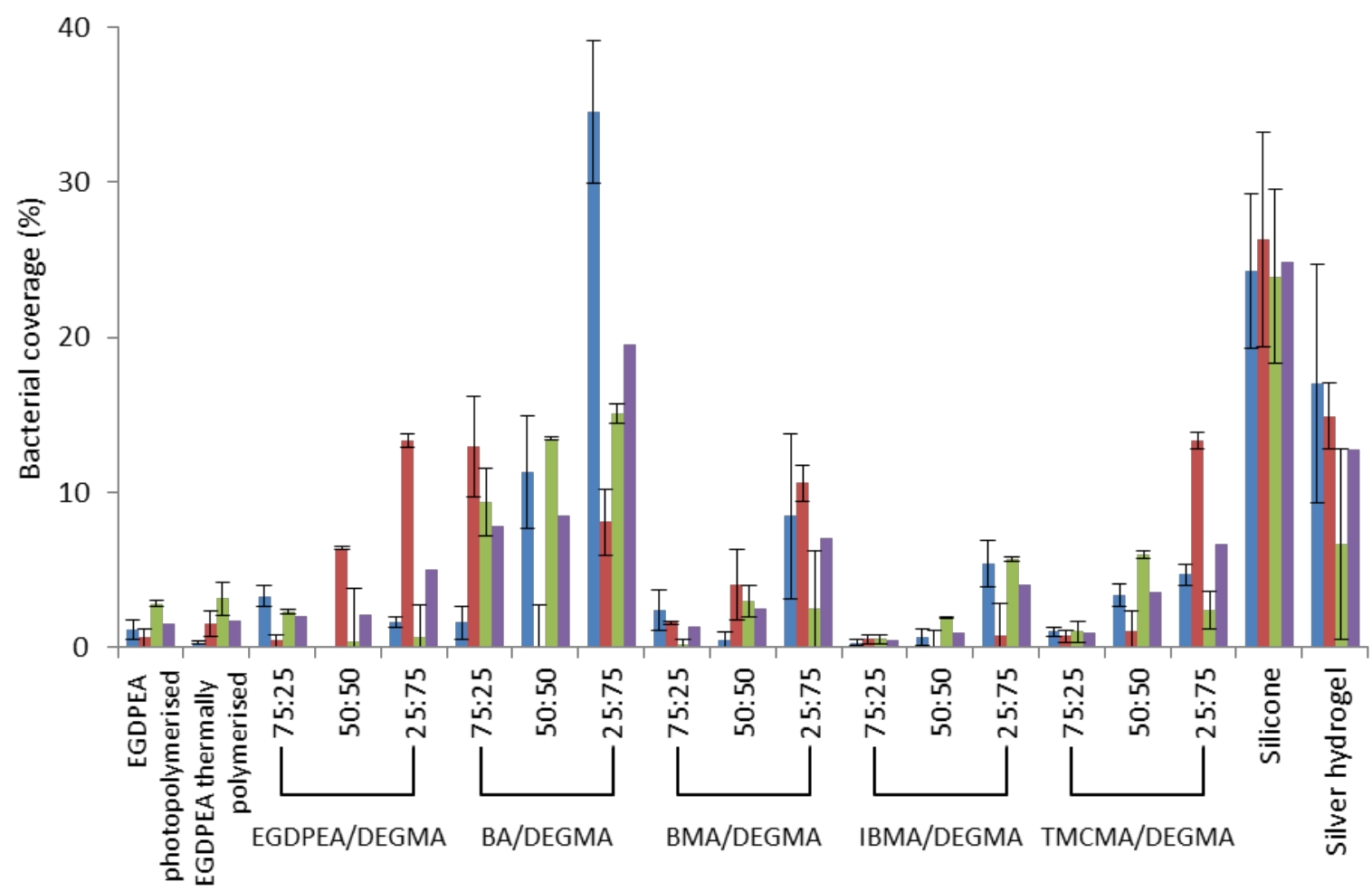

TOC Document for the Manuscript "Application of Targeted Molecular and Material Property Optimization to Bacterial Attachment-Resistant (Meth)acrylate Polymers"

Kevin Adlington, ${ }^{\mathrm{a}}$ Nam T. Nguyen, ${ }^{\mathrm{a}, \mathrm{b}}$ Elizabeth Eaves, ${ }^{\mathrm{a}, \mathrm{b}}$ Jing Yang, ${ }^{\mathrm{c}}$ Chien-Yi Chang, ${ }^{\mathrm{c}, \mathrm{d}}$ Jianing Li, ${ }^{\mathrm{a}, \mathrm{b}}$ Alexandra L. Gower, ${ }^{\mathrm{a}}$ Amy Stimpson, ${ }^{\mathrm{a}}$ Daniel G. Anderson, ${ }^{\mathrm{e}}$ Robert Langer, ${ }^{\mathrm{e}}$ Martyn C. Davies, ${ }^{\mathrm{f}}$ Andrew L. Hook, ${ }^{\mathrm{f}}$ Paul Williams, ${ }^{\mathrm{c}}$ Morgan R. Alexander, ${ }^{\mathrm{f}}$ and Derek J. Irvine $^{\mathrm{a}^{*}}$

${ }^{a}$ Department of Chemical and Environmental Engineering, Faculty of Engineering, University of Nottingham, Nottingham, NG7 2RD, UK.

${ }^{\mathrm{b}}$ School of Chemistry, University of Nottingham, Nottingham, NG7 2RD, UK.

${ }^{c}$ Centre for Biomolecular Sciences and School of Life Sciences, School of Chemistry, University of Nottingham, Nottingham, NG7 2RD, UK.

${ }^{\mathrm{d}}$ Current address - School of Life Sciences, Heriot-Watt University, Edinburgh, EH14 4AS, UK.

${ }^{\mathrm{e}}$ Department of Chemical Engineering, Institute for Medical Engineering and Science, Harvard-MIT Division of Health Sciences and Technology, David H. Koch Institute for Integrative Cancer Research, Massachusetts Institute of Technology, 500 Main Street, Cambridge, MA, 02139, USA.

${ }^{\mathrm{f}}$ Laboratory of Biophysics and Surface Analysis, School of Pharmacy, University of Nottingham, Nottingham, NG7 2RD, UK.

Bacterial coverage of three different strains after $72 \mathrm{~h}$ culture where $\mathrm{PA}=P$. aeruginosa, PAO1 (blue/first bar), SA = S. aureus 8325-4, (red/second bar), UPEC = uropathogenic $E$. coli 536 (green/third bar). Average bacterial attachment across the three different strains (purple/fourth bar). 